\title{
Satisfiability and computing van der Waerden numbers*
}

\author{
Michael R. Dransfield \\ National Security Agency \\ Information Assurance Directorate \\ Ft. Meade, MD 20755 \\ Victor W. Marek \\ Department of Computer Science \\ University of Kentucky \\ Lexington, KY 40506-0046
}

\author{
Lengning Liu \\ Department of Computer Science \\ University of Kentucky \\ Lexington, KY 40506-0046 \\ Mirosław Truszczyński \\ Department of Computer Science \\ University of Kentucky \\ Lexington, KY 40506-0046
}

Submitted: Oct 23, 2002; Accepted: Jun 10, 2004; Published: Jun 16, 2004

MR Subject Classifications: 05D10

\begin{abstract}
In this paper we bring together the areas of combinatorics and propositional satisfiability. Many combinatorial theorems establish, often constructively, the existence of positive integer functions, without actually providing their closed algebraic form or tight lower and upper bounds. The area of Ramsey theory is especially rich in such results. Using the problem of computing van der Waerden numbers as an example, we show that these problems can be represented by parameterized propositional theories in such a way that decisions concerning their satisfiability determine the numbers (function) in question. We show that by using general-purpose complete and local-search techniques for testing propositional satisfiability, this approach becomes effective - competitive with specialized approaches. By following it, we were able to obtain several new results pertaining to the problem of computing van der Waerden numbers. We also note that due to their properties, especially their structural simplicity and computational hardness, propositional theories that arise in this research can be of use in development, testing and benchmarking of SAT solvers.
\end{abstract}

\section{Introduction}

In this paper we discuss how the areas of propositional satisfiability and combinatorics can help advance each other. On one hand, we show that recent dramatic improvements

${ }^{*}$ This is an expanded and updated version of the conference paper [4]. 
in the efficiency of SAT solvers and their extensions make it possible to obtain new results in combinatorics simply by encoding problems as propositional theories, and then computing their models (or deciding that none exist) using off-the-shelf general-purpose SAT solvers. On the other hand, we argue that combinatorics is a rich source of structured, parameterized families of hard propositional theories, and can provide useful sets of benchmarks for developing and testing new generations of SAT solvers.

In our paper we focus on the problem of computing van der Waerden numbers. The celebrated van der Waerden theorem [22] asserts that for every positive integers $k$ and $l$ there is a positive integer $m$ such that every partition of $\{1, \ldots, m\}$ into $k$ blocks (parts) has at least one block with an arithmetic progression of length $l$. The problem is to find the least such number $m$. This number is called the van der Waerden number $W(k, l)$. Exact values of $W(k, l)$ are known only for five pairs $(k, l)$. For other combinations of $k$ and $l$ there are some general lower and upper bounds but they are very coarse and do not give any good idea about the actual value of $W(k, l)$. In the paper we show that SAT solvers such as POSIT [7], and SATO [23], as well as recently developed local-search solver wsat(cc) [14], designed to compute models for propositional theories extended by cardinality atoms [5], can improve lower bounds for van der Waerden numbers for several combinations of parameters $k$ and $l$.

Theories that arise in these investigations are determined by the two parameters $k$ and $l$. Therefore, they show a substantial degree of structure and similarity. Moreover, as $k$ and $l$ grow, these theories quickly become very hard. This hardness is only to some degree an effect of the growing size of the theories. For the most part, it is the result of the inherent difficulty of the combinatorial problem in question. All this suggests that theories resulting from hard combinatorial problems defined in terms of tuples of integers may serve as benchmark theories in experiments with SAT solvers.

There are other results similar in spirit to the van der Waerden theorem. The Schur theorem states that for every positive integer $k$ there is an integer $m$ such that every partition of $\{1, \ldots, m\}$ into $k$ blocks contains a block that is not sum-free. Similarly, the Ramsey theorem (which gave name to this whole area in combinatorics) [18] concerns the existence of monochromatic cliques in edge-colored graphs, and the Hales-Jewett theorem [12] concerns the existence of monochromatic lines in colored cubes. Each of these results gives rise to a particular function defined on pairs or triples of integers and determining the values of these functions is a major challenge for combinatorialists. In all cases, only few exact values are known and lower and upper estimates are very far apart. Many of these results were obtained by means of specialized search algorithms highly depending on the combinatorial properties of the problem (although, we know of no such computational approaches in the context of the van der Waerden numbers). Our paper shows that generic SAT solvers are maturing to the point where they are capable of generating new results for hard combinatorial problems. That combined together with the ease with which they can be used in experimentation (almost no code development time) makes them a useful tool in combinatorial research. 


\begin{tabular}{||lr|r|r|r||}
\hline$k$ & $l$ & 3 & 4 & 5 \\
\hline 2 & & 9 & 35 & 178 \\
3 & & 27 & & \\
4 & & 76 & & \\
\hline
\end{tabular}

Table 1: Known non-trivial values of van der Waerden numbers

\section{2 van der Waerden numbers}

In the paper we use the following terminology. By $\mathbb{Z}^{+}$we denote the set of positive integers and, for $m \in \mathbb{Z}^{+},[m]$ is the set $\{1, \ldots, m\}$. A partition of a set $X$ is a sequence $\mathcal{A}=\left\langle A_{1}, \ldots, A_{k}\right\rangle$ of mutually disjoint subsets of $X$ such that $\cup \mathcal{A}=X$. Elements of $\mathcal{A}$ are commonly called blocks. We note that we deviate here from the standard definition of a partition of a set $X$ as a collection of nonempty and mutually disjoint subsets covering all elements of $X$. From the perspective of van der Waerden numbers, both definitions are equivalent. However, the definition we use here is better aligned with propositional theories we develop later in the paper to model the problem of computing van der Waerden numbers.

Informally, the van der Waerden theorem [22] states that if a sufficiently long initial segment of positive integers is partitioned into a few blocks, then one of these blocks has to contain an arithmetic progression of a desired length. Formally, the theorem is usually stated as follows.

Theorem 2.1 (van der Waerden theorem) For every $k, l \in \mathbb{Z}^{+}$, there exists $m \in \mathbb{Z}^{+}$ such that for every partition $\left\langle A_{1}, \ldots, A_{k}\right\rangle$ of $[m]$, there is $i, 1 \leq i \leq k$, such that block $A_{i}$ contains an arithmetic progression of length at least $l$.

We define the van der Waerden number $W(k, l)$ to be the least number $m$ for which the assertion of Theorem 2.1 holds. Theorem 2.1 states that van der Waerden numbers are well defined.

One can show that for every $k$ and $l$, where $l \geq 2, W(k, l)>k$. In particular, it is easy to see that $W(k, 2)=k+1$. From now on, we focus on the non-trivial case when $l \geq 3$.

Little is known about the numbers $W(k, l)$. In particular, no closed formula has been identified so far and only five exact values are known. They are shown in Table $1[1,11]$.

Since we know few exact values for van der Waerden numbers, it is important to establish good estimates. One can show that the Hales-Jewett theorem entails the van der Waerden theorem, and some upper bounds for the numbers $W(k, l)$ can be derived from the Shelah's proof of the former [20]. Recently, Gowers [10] presented stronger upper bounds, which he derived from his proof of the Szemerédi theorem [21] on arithmetic progressions. 
In our work, we focus on lower bounds. Several general results are known. For instance, Erdös and Rado [6] provided a non-constructive proof for the inequality

$$
W(k, l)>\left(2(l-1) k^{l-1}\right)^{1 / 2} .
$$

For some special values of parameters $k$ and $l$, Berlekamp obtained better bounds by using properties of finite fields [2]. These bounds are still rather weak. His strongest result concerns the case when $k=2$ and $l-1$ is a prime number. Namely, he proved that when $l-1$ is a prime number,

$$
W(2, l)>(l-1) 2^{l-1} .
$$

In particular, $W(2,6)>160$ and $W(2,8)>896$.

Our goal in this paper is to employ propositional satisfiability solvers to find lower bounds for several small van der Waerden numbers. The bounds we find significantly improve on the ones implied by the results of Erdös and Rado, and Berlekamp.

We proceed as follows. For each triple of positive integers $\langle k, l, m\rangle$, we define a propositional CNF theory $\operatorname{vdW}_{k, l, m}$ and then show that $\mathrm{vdW}_{k, l, m}$ is satisfiable if and only if $W(k, l)>m$. With such encodings, one can use SAT solvers (at least in principle) to determine the satisfiability of $\mathrm{vdW}_{k, l, m}$ and, consequently, find $W(k, l)$. Since $W(k, l)>k$, without loss of generality we can restrict our attention to $m>k$. We also show that more concise encodings are possible, leading ultimately to better bounds, if we use an extension of propositional logic by cardinality atoms and apply to them solvers capable of handling such atoms directly.

To describe $\mathrm{vdW}_{k, l, m}$ we will use a standard first-order language, without function symbols, but containing a predicate symbol in_block and constants $1, \ldots, m$. An intuitive reading of a ground atom in_block $(i, b)$ is that an integer $i$ is in block $b$.

We now define the theory $\mathrm{vdW}_{k, l, m}$ by including in it the following clauses:

$\operatorname{vdW1:~} \neg i n_{\_} b l o c k\left(i, b_{1}\right) \vee \neg i n_{-} b l o c k\left(i, b_{2}\right)$, for every $i \in[m]$ and every $b_{1}, b_{2} \in[k]$ such that $b_{1}<b_{2}$,

$\operatorname{vdW} 2: \quad i n_{-} b l o c k(i, 1) \vee \ldots \vee$ in_block $(i, k)$, for every $i \in[m]$,

vdW3: $\neg$ in_block $(i, b) \vee \neg i n_{-} b l o c k(i+d, b) \vee \ldots \vee \neg i n_{-} b l o c k(i+(l-1) d, b)$, for every $i, d \in[m]$ such that $i+(l-1) d \leq m$, and for every $b$ such that $1 \leq b \leq k$.

As an aside, we note that we could design $\mathrm{vdW}_{k, l, m}$ strictly as a theory in propositional language using propositional atoms of the form $i n_{-} b l o c k_{i, b}$ instead of ground atoms in_block $(i, b)$. However, our approach opens a possibility to specify this theory as finite (and independent of data) collections of propositional schemata, that is, open clauses in the language of first-order logic without function symbols. Given a set of appropriate constants (to denote integers and blocks) such theory, after grounding, coincides with $\mathrm{vdW}_{k, l, m}$. In fact, we have defined an appropriate syntax that allows us to specify both data and schemata and implemented a grounding program psgrnd [5] that generates their equivalent ground (propositional) representation. This grounder accepts arithmetic expressions as well as simple relational expressions (equalities and comparisons), and 
evaluates and eliminates them according to their standard interpretation. Such approach significantly simplifies the task of developing propositional theories that encode problems, as well as the use of SAT solvers [5].

Propositional interpretations of the theory $\mathrm{vdW}_{k, l, m}$ can be identified with subsets of the set of atoms $\{$ in_block $(i, b): i \in[m], b \in[k]\}$. Namely, a set $M \subseteq\{$ in_block $(i, b): i \in$ $[m], b \in[k]\}$ determines an interpretation in which all atoms in $M$ are true and all other atoms are false. In the paper we always assume that interpretations are represented as sets.

It is easy to see that clauses (vdW1) ensure that if $M$ is a model of $\mathrm{vdW}_{k, l, m}$ (that is, is an interpretation satisfying all clauses of $\left.\mathrm{vdW}_{k, l, m}\right)$, then for every $i \in[m], M$ contains at most one atom of the form $i n_{-} b l o c k(i, b)$. Clauses (vdW2) ensure that for every $i \in[m]$ there is at least one $b \in[k]$ such that $i n_{-} b \operatorname{lock}(i, b) \in M$. In other words, clauses (vdW1) and (vdW2) together ensure that if $M$ is a model of $\mathrm{vdW}_{k, l, m}$, then $M$ determines a partition of $[m]$ into $k$ blocks.

The last group of constraints, clauses (vdW3), guarantee that elements from $[m]$ forming an arithmetic progression of length $l$ do not all belong to the same block. All these observations imply the following result.

Proposition 2.2 There is a one-to-one correspondence between models of the formula $v d W_{k, l, m}$ and partitions of $[\mathrm{m}]$ into $k$ blocks so that no block contains an arithmetic progression of length $l$. Specifically, an interpretation $M$ is a model of $v d W_{k, l, m}$ if and only if $\langle\{i \in[m]:$ in_block $(i, b) \in M\}: b \in[k]\rangle$ is a partition of $[m]$ into $k$ blocks such that no block contains an arithmetic progression of length $l$.

Proposition 2.2 has the following direct corollary.

Corollary 2.3 For every positive integers $k, l$, and $m$, with $l \geq 2$ and $m>k, m<$ $W(k, l)$ if and only if the formula $v d W_{k, l, m}$ is satisfiable.

It is evident that if $m$ has the property that $\mathrm{vdW}_{k, l, m}$ is unsatisfiable then for every $m^{\prime}>m, \mathrm{vdW}_{k, l, m^{\prime}}$ is also unsatisfiable. Thus, Corollary 2.3 suggests the following algorithm that, given $k$ and $l$, computes the van der Waerden number $W(k, l)$ : for consecutive integers $m=k+1, k+2, \ldots$ we test whether the theory $\mathrm{vdW}_{k, l, m}$ is satisfiable. If so, we continue. If not, we return $m$ and terminate the algorithm. By the van der Waerden theorem, this algorithm terminates.

It is also clear that there are simple symmetries in the van der Waerden problem. If a set $M$ of atoms of the form in_block $(i, b)$ is a model of the theory $\mathrm{vdW}_{k, l, m}$, and $\pi$ is a permutation of $[k]$, then the corresponding set of atoms $\{$ in_block $(i, \pi(b)):$ in_block $(i, b) \in M\}$ is also a model of $\mathrm{vdW}_{k, l, m}$, and so is the set of atoms $\{$ in_block $(m+1-i, b):$ in_block $(i, b) \in$ $M\}$.

Following the approach outlined above, adding clauses to break these symmetries, and applying POSIT [7] and SATO [23] as SAT solvers we were able to establish that $W(4,3)=76$ and compute a "library" of counterexamples (partitions with no block 
containing arithmetic progressions of a specified length) for $m=75$. We were also able to find several lower bounds on van der Waerden numbers for larger values of $k$ and $m$.

However, a major limitation of our first approach is that the size of theories $\mathrm{vdW}_{k, l, m}$ grows quickly and makes complete SAT solvers ineffective. Let us estimate the size of the theory $\mathrm{vdW}_{k, l, m}$. The total size of clauses (vdW1) (measured as the number of atom occurrences) is $\Theta\left(m k^{2}\right)$. The size of clauses (vdW2) is $\Theta(m k)$. Finally, the size of clauses (vdW3) is $\Theta\left(m^{2}\right)$ (indeed, there are $\Theta\left(m^{2} / l\right)$ arithmetic progressions of length $l$ in $\left.[m]\right)^{1}$. Thus, the total size of the theory $\mathrm{vdW}_{k, l, m}$ is $\Theta\left(m k^{2}+m^{2}\right)$.

To overcome this obstacle, we used a two-pronged approach. First, as a modeling language we used PS+ logic [5], which is an extension of propositional logic by cardinality atoms. Cardinality atoms support concise representations of constraints of the form "at least $p$ and at most $r$ elements in a set are true" and result in theories of smaller size. Second, we used a local-search algorithm, wsat(cc), for finding models of theories in logic PS + that we have designed and implemented recently [14]. Using encodings as theories in logic PS+ and wsat(cc) as a solver, we were able to obtain substantially stronger lower bounds for van der Waerden numbers than those known to date.

We will now describe this alternative approach. For a detailed treatment of the PS+ logic we refer the reader to [5]. In this paper, we will only review most basic ideas underlying the logic PS+ (in its propositional form). Let $A t$ be a set of propositional atoms. By a propositional cardinality atom (c-atom for short), we mean any expression of the form $m\left\{p_{1}, \ldots, p_{k}\right\} n$ (one of $m$ and $n$, but not both, may be missing), where $m$ and $n$ are non-negative integers and $p_{1}, \ldots, p_{k}$ are propositional atoms from $A t$. The notion of a clause generalizes in an obvious way to the language with cardinality atoms. Namely, a c-clause is an expression of the form

$$
C=A_{1} \vee \ldots \vee A_{s} \vee \neg B_{1} \vee \ldots \vee \neg B_{t},
$$

where all $A_{i}$ and $B_{i}$ are (propositional) atoms or cardinality atoms.

Let $M \subseteq A t$ be a set of atoms. We say $M$ satisfies a cardinality atom $m\left\{p_{1}, \ldots, p_{k}\right\} n$ if

$$
m \leq\left|M \cap\left\{p_{1}, \ldots, p_{k}\right\}\right| \leq n .
$$

If $m$ is missing, we only require that $\left|M \cap\left\{p_{1}, \ldots, p_{k}\right\}\right| \leq n$. Similarly, when $n$ is missing, we only require that $m \leq\left|M \cap\left\{p_{1}, \ldots, p_{k}\right\}\right|$. A set of atoms $M$ satisfies a c-clause $C$ of the form (1) if $M$ satisfies at least one atom $A_{i}$ or does not satisfy at least one atom $B_{j}$. We note that the expression $1\left\{p_{1}, \ldots, p_{k}\right\} 1$ expresses the quantifier "There exists exactly one ..." - commonly used in mathematical statements.

It is now clear that all clauses (vdW1) and (vdW2) from $\mathrm{vdW}_{k, l, m}$ can be represented in a more concise way by the following collection of c-clauses:

$\operatorname{vdW}^{\prime} 1: \quad 1\left\{\operatorname{in\_ } b l o c k(i, 1), \ldots\right.$, in_block $\left.(i, k)\right\} 1$, for every $i \in[m]$.

Indeed, c-clauses (vdW'1) enforce that their models, for every $i \in[m]$ contain exactly one atom of the form in_block $(i, b)$ - precisely the same effect as that of clauses (vdW1)

\footnotetext{
${ }^{1}$ Goldstein [9] provided a precise formula. When $r=(m-1)-(l-1)\left\lfloor\frac{m-1}{l-1}\right\rfloor$ and $q=\left\lfloor\frac{m-1}{l-1}\right\rfloor$ then there are $q \cdot r+\left(\begin{array}{c}q-1 \\ 2\end{array}\right) \cdot(l-1)$ arithmetic progressions of length $l$ in $[m]$.
} 
and (vdW2). Let $\mathrm{vdW}_{k, l, m}^{\prime}$ be a PS+ theory consisting of clauses (vdW'1) and (vdW3). It follows that Proposition 2.2 and Corollary 2.3 can be reformulated by replacing vdW $\mathrm{W}_{k, l, m}$ with vdW $\mathrm{vd}_{k, l, m}^{\prime}$ in their statements. Consequently, any algorithm for finding models of PS+ theories can be used to compute van der Waerden numbers (or, at least, some bounds for them) in the way we described above.

The adoption of cardinality atoms leads to a more concise representation of the problem. While, as we discussed above, the size of all clauses (vdW1) and (vdW2) is $\Theta\left(m k^{2}+m k\right)$, the size of clauses ( $\left.\mathrm{vdW}^{\prime} 1\right)$ is $\Theta(m k)$.

\section{Computing models of theories vdW $\mathbf{W}_{k, l, m}^{\prime}$}

As we noted earlier, to compute models of theories $\mathrm{vdW}_{k, l, m}$ (no c-atoms) we used complete solvers POSIT and SATO. They were only practical for our experiments with the theory $\mathrm{vdW}_{4,3, m}$. For the most part, we were working with theories $\mathrm{vdW}_{k, l, m}^{\prime}$ and to compute their models we used the local-search algorithm wsat(cc) [14], extended with bootstrapping [15]. Wsat(cc) is based on the same ideas as wsat [19]. The search consists of $t$ tries, each starting in a complete truth assignment, called an initial truth assignment (ITA, for short), and proceeding in a sequence of $f$ local improvement steps, called flips. A major difference is that due to the presence of c-atoms in c-clauses, wsat(cc) uses different formulas to calculate the breakcount and proposes several other heuristics designed specifically to handle c-atoms.

Wsat(cc) is an incomplete solver and it does not guarantee that it can find a solution when there is one. The likelihood that a try terminates with the success depends on the proximity of an ITA used in the try to a satisfying truth assignment. It is a non-trivial problem to generate "good" ITA's. In [15], we proposed and implemented a bootstrapping technique to address it. We call a theory $T^{\prime}$ a relaxation of a theory $T$ if for every model $M$ of $T, M \cap A t\left(T^{\prime}\right)$ is a model of $T^{\prime}$. Given a theory $T$ and its relaxation $T^{\prime}$, The bootstrapping consists of using satisfying assignments for $T^{\prime}$ as ITAs in tries when searching for satisfying assignments for $T$. The underlying intuition is that a relaxation of a theory is easier to solve than the theory itself and that solutions to $T^{\prime}$ are more likely to be close to solutions to $T$ than random assignments.

To search for models of theories $\mathrm{vdW}_{k, l, m}^{\prime}$, we used wsat(cc) combined with bootstrapping. Our approach exploited the fact that if $m^{\prime}<m$, then the theory $\mathrm{vdW}_{k, l, m^{\prime}}^{\prime}$ is a relaxation of the theory $\mathrm{vdW}_{k, l, m}^{\prime}$. Indeed, for every partition of $[m]$ into $k$ blocks so that none of the blocks contains an arithmetic progression of length $l$, the restriction of this partition to $\left[\mathrm{m}^{\prime}\right]$ is a partition of $\left[\mathrm{m}^{\prime}\right]$ into $k$ blocks, none of which contains an arithmetic progression of length $l$. That observation, expressed in terms of models of theories $\mathrm{vdW}_{k, l, m}^{\prime}$ and $\mathrm{vdW}_{k, l, m^{\prime}}^{\prime}$ directly implies the claim.

In its implementation, bootstrapping uses a sequence of relaxations. To compute models of $\mathrm{vdW}_{k, l . m}$, we construct a sequence of relaxations: $\mathrm{vdW}_{k, l, m_{1}}, \ldots, \mathrm{vdW}_{k, l, m_{k}}$, where $m_{1}<\ldots<m_{k}=m$. Given that sequence, the algorithm proceeds as follows:

1. it starts at level 1 and uses wsat(cc) with randomly generated ITAs to find models 
for the first theory in the sequence, $\mathrm{vdW}_{k, l, m_{1}}$;

2. each time the algorithm finds a model $S$ for a theory $\mathrm{vdW}_{k, l, m_{i}}$, it moves to the next theory in the sequence, $\mathrm{vdW}_{k, l, m_{i+1}}$, and runs wsat(cc) on $\mathrm{vdW}_{k, l, m_{i+1}}$ with the truth assignment given by $S$ as an ITA (we randomly extend $S$ to a complete assignment for the language of the theory $\left.\mathrm{vdW}_{k, l, m_{i+1}}\right)$;

3. if at any level $i$, wsat(cc) fails to find models, the algorithm restarts computation from level 1;

4. if the algorithm finds a model at level $k$ (for the theory $\mathrm{vdW}_{k, l, m}^{\prime}$ ), the algorithm stops and outputs the model. Moreover, $m$ is a lower bound for the van der Waerden number $W(k, l)$.

\section{Results}

Our goal is to establish lower bounds for small van der Waerden numbers by exploiting propositional satisfiability solvers. Here is a summary of our results.

1. Using complete SAT solvers POSIT and SATO and the encoding of the problem as $\mathrm{vdW}_{k, l, m}$, we found a "library" of all counterexamples to the fact that $W(4,3)=75$. Up to obvious symmetries - permutations of blocks and the "reflection" symmetry $i \mapsto m+1-i$ - there are 30 of them. We list two of them in the appendix. A complete list can be found at http://www.cs.uky.edu/ai/vdw/. By inspecting all partitions in the library, one can see that applying the "reflection" symmetry never leads to the same result as applying a "block-permutation" symmetry. It is also easy to see that the "reflection" symmetry commutes with every "block-permutation" symmetry. It follows from these two observations, that the cardinality of the orbit of each of the library partitions is 48 . Consequently, the full list of counterexample partitions consists of 1440 elements.

2. We found that the formula $\mathrm{vdW}_{4,3,76}$ is unsatisfiable. Hence, we found that a "generic" SAT solver is capable of finding that $W(4,3)=76$.

3. We established several new lower bounds for the numbers $W(k, l)$. They are presented in Table 2. Partitions demonstrating that $W(2,8)>1322, W(3,5)>676$, and $W(4,4)>416$ are included in the appendix. All up-to-date results on the lower bounds on van der Waerden numbers are available at http://www.cs.uky . edu/ai/ vdw/ (we are continually running our local-search solver and update the bounds as we improve on them). To the best of our knowledge there have been no published results on lower bounds for the unknown van der Waerden numbers other than those that follow from the formula of Erdös and Rado [6] and (restricted to only some combinations of $k$ and $l$ ) a stronger formula implied by the result of Berlekamp [2]. Our lower bounds are first results obtained through computer calculations and they significantly improve on the values implied by the two formulas mentioned above. 
Table 2: Extended results on van der Waerden numbers

\begin{tabular}{|c|c|c|c|c|c|c|}
\hline$k^{l}$ & 3 & 4 & 5 & 6 & 7 & 8 \\
\hline 2 & 9 & 35 & 178 & $>341$ & $>614$ & $>1322$ \\
\hline 3 & 27 & $>193$ & $>676$ & $>2236$ & & \\
\hline 4 & 76 & $>416$ & & & & \\
\hline 5 & $>125$ & $>880$ & & & & \\
\hline 6 & $>194$ & & & & & \\
\hline
\end{tabular}

Table 3: Numbers of atoms and clauses in theories $\mathrm{vdW}_{k, l, m}^{\prime}$, used to establish the results presented in Table 2.

\begin{tabular}{|c|c|c|c|c|c|c|}
\hline$k \quad l$ & 3 & 4 & 5 & 6 & 7 & 8 \\
\hline 2 & $\begin{array}{l}18 \\
41\end{array}$ & $\begin{array}{r}70 \\
409\end{array}$ & $\begin{array}{r}356 \\
7922\end{array}$ & $\begin{array}{r}682 \\
23257\end{array}$ & $\begin{array}{r}1228 \\
23834\end{array}$ & $\begin{array}{r}2644 \\
249670\end{array}$ \\
\hline 3 & $\begin{array}{l}108 \\
534\end{array}$ & $\begin{array}{r}579 \\
18529\end{array}$ & $\begin{array}{r}2028 \\
171028\end{array}$ & $\begin{array}{r}6708 \\
1498792\end{array}$ & & \\
\hline 4 & $\begin{array}{r}304 \\
5700\end{array}$ & $\begin{array}{r}1664 \\
114956\end{array}$ & & & & \\
\hline 5 & $\begin{array}{r}625 \\
19345\end{array}$ & $\begin{array}{r}4400 \\
644015\end{array}$ & & & & \\
\hline 6 & $\begin{array}{r}1164 \\
56066\end{array}$ & & & & & \\
\hline
\end{tabular}

To provide some insight into the complexity of the satisfiability problems involved, in Table 3 we list the number of atoms and the number of clauses in the theories $\mathrm{vdW}_{k, l, m}^{\prime}$. Specifically, the entry $k, l$ in this table contains the number of atoms and the number of clauses in the theories $\mathrm{vdW}_{k, l, m}^{\prime}$, where $m$ is the value given in the entry $k, l$ in Table 2 .

\section{Discussion}

Recent progress in the development of SAT solvers provides an important tool for researchers looking for both the existence and non-existence of various combinatorial objects. We have demonstrated that several classical questions related to van der Waerden numbers can be naturally cast as questions on the existence of satisfying valuations for some propositional CNF-formulas.

Computing combinatorial objects such as van der Waerden numbers is hard. They 
are structured but as we pointed out few values are known, and new results are hard to obtain. Thus, the computation of those numbers can serve as a benchmark ('can we find the configuration such that...') for complete and local-search methods, and as a challenge ('can we show that a configuration such that ...' does not exist) for complete SAT solvers. Moreover, with powerful SAT solvers it is likely that the bounds obtained by computation of counterexamples are "sharp" in the sense that when a configuration is not found then none exist. For instance it is likely that $W(5,3)$ is close to 126 (possibly, it is 126), because 125 was the last integer where we were able to find a counterexample despite significant computational effort. This claim is further supported by the fact that in all examples where exact values are known, our local-search algorithm was able to find counterexample partitions for the last possible value of $m$. The lower-bounds results of this sort may constitute an important clue for researchers looking for nonexistence arguments and, ultimately, for the closed form of van der Waerden numbers.

A major impetus for the recent progress of SAT solvers comes from applications in computer engineering. In fact, several leading SAT solvers such as zCHAFF [17] and berkmin [8] have been developed with the express goal of aiding engineers in correctly designing and implementing digital circuits. Yet, the fact that these solvers are able to deal with hard optimization problems in one area (hardware design and verification) carries the promise that they will be of use in another area - combinatorial optimization. Our results indicate that it is likely to be the case.

The current capabilities of SAT solvers has allowed us to handle large instances of these problems. Better heuristics and other techniques for pruning the search space will undoubtedly further expand the scope of applicability of generic SAT solvers to problems that, until recently, could only be solved using specialized software.

\section{Acknowledgments}

This research has been supported by the Center for Communication Research, La Jolla and by the NSF grants IIS-0097278 and IIS-0325063.

\section{References}

[1] M.D. Beeler and P.E. O'Neil. Some new van der Waerden numbers, Discrete Mathematics, 28:135-146, 1979.

[2] E. Berlekamp. A construction for partitions which avoid long arithmetic progressions. Canadian Mathematical Bulletin 11:409-414, 1968.

[3] M. Davis and H. Putnam. A computing procedure for quantification theory, Journal of the Association for Computing Machinery, 7:201-215, 1960.

[4] M.R. Dransfield, V.M. Marek and M. Truszczyński. Satisfiability and computing van der Waerden numbers, in Theory and Applications of Satisfiability Testing, 6th In- 
ternational Conference, SAT-2003, Lecture Notes in Computer Science, 2919, pages 1-13, Springer, 2001.

[5] D. East and M. Truszczyński. Predicate-calculus based logics for modeling and solving search problems. ACM Transactions on Computational Logic, To appear, available at http://www.acm.org/tocl/accepted.html, 2005.

[6] P. Erdös and R. Rado. Combinatorial theorems on classifications of subsets of a given set, Proceedings of London Mathematical Society, 2:417-439, 1952.

[7] J.W. Freeman. Improvements to propositional satisfiability search algorithms, PhD thesis, Department of Computer Science, University of Pennsylvania, 1995.

[8] E. Goldberg, Y. Novikov. BerkMin: a Fast and Robust SAT-Solver. DATE-2002, pages 142-149, 2002.

[9] D. Goldstein. Personal communication, 2002.

[10] T. Gowers. A new proof of Szemerédi theorem. Geometric and Functional Analysis, 11:465-588, 2001.

[11] R.L. Graham, B.L. Rotschild, and J.H. Spencer. Ramsey Theory, Wiley, 1990.

[12] A. Hales and R.I. Jewett. Regularity and positional games, Transactions of American Mathematical Society, 106:222-229, 1963.

[13] R.E. Jeroslaw and J. Wang. solving propositional satisfiability problems, Annals of Mathematics and Artificial Intelligence, 1:167-187, 1990.

[14] L. Liu and M. Truszczyński. Local-search techniques in propositional logic extended with cardinality atoms, Proceedings of the 9th International Conference on Principles and Practice of Constraint Programming, CP-2003, Lecture Notes in Computer Science, vol. 2833, 495-509, Springer, 2003.

[15] L. Liu and M. Truszczyński. Local-search with bootstrapping. In Proceedings of SAT 2004, Vancouver, Canada, 2004.

[16] J.P. Marques-Silva and K.A. Sakallah. GRASP: A new search algorithm for satisfiability, IEEE Transactions on Computers, 48:506-521, 1999.

[17] M.W. Moskewicz, C.F. Magidan, Y. Zhao, L. Zhang, and S. Malik. Chaff: engineering an efficient SAT solver, in SAT 2001, 2001.

[18] F.P. Ramsey. On a problem of formal logic, Proceedings of London Mathematical Society, 30:264-286, 1928.

[19] B. Selman, H.A. Kautz, and B. Cohen. Noise Strategies for Improving Local Search. Proceedings of AAAI'94, pp. 337-343. MIT Press 1994. 
[20] S. Shelah. Primitive recursive bounds for van der Waerden numbers, Journal of American Mathematical Society, 1:683-697, 1988.

[21] E. Szemerédi. On sets of integers containing no $k$ elements in arithmetic progression, Acta Arithmetica, 27:199-243, 1975.

[22] B.L. van der Waerden. Beweis einer Baudetschen Vermutung, Nieuwe Archief voor Wiskunde, 15:212-216, 1927.

[23] H. Zhang. SATO: An efficient propositional prover, in Proceedings of CADE-17, pages 308-312, 1997. Springer Lecture Notes in Artificial Intelligence 1104.

\section{Appendix}

Using a complete SAT solver we computed the library of all partitions (up to isomorphism) of [75] showing that $75<W(4,3)$. Two of these 30 partitions are shown below:

Solution 1:

Block 1: 6791418202324363843444651555760617375

Block 2: 4512222628293137414249596365666874

Block 3: 12810111317273435394547485054647172

Block 4: 31516192125303233405253565862676970

Solution 2:

Block 1: 67914182023243638434446515557606173

Block 2: 4512222628293137414249596365666874

Block 3: 12810111317273435394547485054647172

Block 4: 3151619212530323340525356586267697075

These two and the remaining 28 partitions can be found at http://www.cs.uky.edu/ai/vdw/

Next, we exhibit a partition of [1322] into two blocks demonstrating that $W(2,8)>1322$.

Block 1:

123456791112161920212225272931323740414243444652535456586365 666769707273798182838485878889909294969798100103104106109110114 116117118120127129131133136138142143146147149150154155159161163167168 172173175176178185186188189190191193194195196197201202206209211213216 218222224225227228229234236237238239240241242248252253257259260261264 265266268269270272273275276277278279280282283285291292293294297298299 300302306312313314316318321322324326327330332334338339340343345347352 355356357358359360362364365367369371372375380382383384386387388389391 395396398405409410411415419423424425426427429430433435436440444445447 448452453454460466467468469472475477479481482483484485487490491492494 496500501502503504505507509510511513514519521522523 ! 527529530531533 
534536537538539540541542544547549550552553554555556560565566568570571 572574575576578585586588595601602608612614617620623625627629632633634 635636637638641642644647652654656659660661662667669670671673674675676 677680682685686687689691693695697698700702703705708709710711712715716 718720721722723724725732735736738739743747751753754755758762763764767 769771772773774775776777780781782783786788789791796798799801803811812 813814817818819820823825827828830831832833834835836838844846847854858 861862864867868869870871874876879884885888889890891892893894896897898 900901905906907910911912913914915916918923925926927928930931932934936 938939941942943948953955959960961969970971972973977980981983985986991 99499599699799910001001100210041006100 ! 810091012101310151016101710181019 1021102210261027 ! 1029103010331038103910411046104710481050105110531054 105610571061106210631065106610681069107110731074107710781082108410851086 108710901092109310961098110211031109111211151117111811221123112511291130 113111331135113711391140114211431144114511471148114911531154115611571163 116611691171117211731175117611801184118611871188119411981199120312041205 120612081210121112121213121612171220122112241227122912301235123612381240 124112431247124812491250125112551256125712581259126212641267126812701273 127512761278128012851286128712881290129112951296129812991301130213041306 13091311131513201321

Block 2:

810131415171823242628303334353638394547484950515557596061626468 717475767778808691939599101102105107108111112113115119121122123124 125126128130132134135137139140141144145148151152153156157158160162164 165166169170171174177179180181182183184187192198199200203204205207208 210212214215217219220221223226230231232233235243244245246247249250251 254255256258262263267271274281284286287288289290295296301303304305307 308309310311315317319320323325328329331333335336337341342344346348349 350351353354361363366368370373374376377378379381385390392393394397399 400401402403404406407408412413414416417418420421422428431432434437438 439441442443446449450451455456457458459461462463464465470471473474476 47848048648848949349549749849950650851251551651751 ! 8520524525526528 532535543545546548551557558559561562563564567569573577579580581582583 584587589590591592593594596597598599600603604605606607609610611613615 616618619621622624626628630631639640643645646648649650651653655657658 663664665666668672678679681683684688690692694696699701704706707713714 717719726727728729730731733734737740741742744745746748749750752756757 759760761765766768770778779784785787790792793794795797800802804805806 807808809810815816821822824826829837839840841842843845848849850851852 853855856857859860863865866872873875877878880881882883886887895899902 903904908909917919920921922924929933935937940944945946947949950951952 954956957958962963964965966967968974975976978979982984987988989990992 9939981003100510071010101110141020102 ! 310241025102810311032103410351036 1037104010421043 ! 1044104510491052105510581059106010641067107010721075 107610791080108110831088108910911094109510971099110011011104110511061107 
110811101111111311141116111911201121112411261127112811321134113611381141 114611501151115211551158115911601161116211641165116711681170117411771178 117911811182118311851189119011911192119311951196119712001201120212071209 121412151218121912221223122512261228123112321233123412371239124212441245 124612521253125412601261126312651266126912711272127412771279128112821283 128412891292129312941297130013031305130713081310131213131314131613171318 13191322

Next, we exhibit a partition of [676] into three blocks demonstrating that $W(3,5)>676$.

Block 1:

2567810111525303132333941434749565862636567717375767787889395 106108109110112118120122125126128129130132133136137138145147150153155 157159166167172173174176178179182183184186187188191197198202205208210 211220231233251252266268273276277278281282286288289291292293297301302 307308310311313315316317318320322323327330331332336340341342345348351 353357359360365369372376377386405411414417419422423425426432434435442 443444446447449451454455457458460461466477480484485486489490492500501 505507508511513515517520521522524530532536541552562563565566567568570 571572577591592598601610616617618622627630632634635636640651653656657 660661662666667672676

\section{Block 2:}

13491213162122262937384446485051545559616466697980828485869192 969798100105107111113116119123131134135141144146149151158161164168170 180181190193194203206207213215216218219221223226227228229234235236238 239241243248250256259260261264270271274275279284285296300304306312319 324325326328334335338339346349355358366367368371373374378379380384387 389391392396398400401406409413416421428430431433436437438440441445450 453456459463465467468470471473479481483491495497499503504510514528531 534535540543544545546549550556558559560561564569574575576580581583584 585586588595599605606608609611613620621625626629638639641643646648649 650659663664665668670671674

\section{Block 3:}

141718192023242728343536404245525357606870727478818389909499101 102103104114115117121124127139140142143148152154156160162163165169171 175177185189192195196199200201204209212214217222224225230232237240242 244245246247249253254255257258262263265267269272280283287290294295298 299303305309314321329333337343344347350352354356361362363364370375381 382383385388390393394395397399402403404407408410412415418420424427429 439448452462464469472474475476478482487488493494496498502506509512516 518519523525526527529533537538539542547548551553554555557573578579582 587589590593594596597600602603604607612614615619623624628631633637642 644645647652654655658669673675 
Finally, we exhibit a partition of [416] into four blocks demonstrating that $W(4,4)>416$.

Block 1:

2711161721242930323941425051576467686976788088919396110122124130 132133134137142148155157159160164165166169172176181182183185194195202 204209212213219243246247248253254255257260264270272276277278280281286 289293303304309310312313317322330336341345347350359361375381383384385 394398399400403404406410411

Block 2:

348131420283135404445525961717982838589929798100101106109117120127 128135140141144146147152154156163168177179189193203208216217222224233 235236244249251256258267268273274275279282284287294295297298300301305 307324326331333338339340348349353356360362365368369370376386387396402408

Block 3:

61518192223434647495455566062636566737577818487102104107111112113 115116125126129136138143158162178180187190191192197201206207210211218 223225226228229237238241242245250252261263265266269271291306308311315 318319321327343344352354355357358363374377378379382388389390392395405 407409412414415416

Block 4:

159101225262733343637384853587072748690949599103105108114118119121 123131139145149150151153161167170171173174175184186188196198199200205 214215220221227230231232234239240259262283285288290292296299302314316 320323325328329332334335337342346351364366367371372373380391393397401413

Configurations showing the validity of other lower bounds listed in Table 2 are available at http: //www.cs.uky.edu/ai/vdw/. 